\title{
Knowledge Market: Agent-mediated Knowledge Sharing
}

\author{
Virginia Dignum $^{1,2}$, Frank Dignum ${ }^{2}$ \\ ${ }^{1}$ Achmea, The Netherlands, \\ email: virginia.dignum@achmea.nl \\ ${ }^{2}$ University Utrecht, The Netherlands, \\ email: \{virginia, dignum\}@cs.uu.nl
}

\begin{abstract}
Like multi-agent systems, KM collaboration environments can be seen as distributed systems where different actors, each pursuing its own goals, need to interact in order to achieve their goals and realize organizational objectives. In this paper, we present a agent-based model for organizations that supports individual initiative and collaboration while prescribing a formal model for organizational processes. This model enables the development of people-oriented KM environments that focus on the collaboration between people. The model is applied to the development of a knowledge market.
\end{abstract}

\section{Introduction}

Recent developments show a shift in the focus of Knowledge Management (KM) from knowledge organization to collaboration. That is, the aim of KM is no longer just the management of activities related to the creation, preservation and distribution of knowledge assets but mainly the management and nurturing of collaboration between people. This is the focus of developments around Community of Practice (CoP) currently taking place at Achmea ${ }^{1}$. A CoP is a group of people who share a common area of expertise and/or who search for solutions to common problems. CoPs are perceived at Achmea as the basic organizational unit of knowledge management and a success factor for sustainable advantage. People in a CoP can perform the same type of job, collaborate on a shared task or work together on a product, however CoPs are not necessarily authorized or identified groups. What holds them together is a common sense of purpose and a real need to know what each other knows. Nurturing communities is hard enough when the members are in a single location with good connectivity and increases considerably when the members are spread around different locations, possibly in different regions, languages and cultures.

Members of distributed communities are not always aware of each other's capabilities and often they will discuss their business problems with a direct colleague just because he/she happens to be conveniently close and not because he/she is the best person to consult with. Links between members of distributed CoPs can be

\footnotetext{
${ }^{1}$ Achmea is one of the largest insurance and financial services company in the Netherlands.
} 
strengthened by webs of communication technologies. This indicates a need for collaboration management systems with the following objectives:

- Assist people generate and apply 'just in time' and 'just enough' knowledge, prevent information overload and stimulate sharing of relevant knowledge in a dynamic, collaborative environment.

- Preserve individual autonomy and contribute to the creation of a atmosphere of trust between participants.

- $\quad$ Provide links individual action and company structure such that on one hand, innovative ways of doing things can be effectively integrated into company processes and, on the other hand, it can be verified whether actions are conform to company values and norms.

There is currently an increasing interest in the use of multi-agent concepts for KM, mainly motivated by the fact that, like multi-agent systems, KM domains involve an inherent distribution of sources, problem solving capabilities and responsibilities. That, is, the integrity of the existing organizational structure and the autonomy of participants must be maintained, which calls for a autonomous and distributed representation of $\mathrm{KM}$ systems. Interactions in $\mathrm{KM}$ environments are fairly sophisticated, including negotiation, information sharing and coordination, and require complex social skills with which agents can be endowed. Furthermore, solutions for KM problems cannot be entirely prescribed from start to finish and therefore reactive and proactive problem solvers are required that can respond to changes in the environment, react to the unpredictability of business process and act on opportunities when they arise.

Agent-based models for KM see agents as autonomous social entities (like employees in a company) that exhibit flexible, responsive and proactive behavior and the interactions among these entities give rise to complex dynamics. We have developed an multi-agent organizational model that attempts to incorporate formal organizational processes and goals and the different individual perspectives of the actors (people, groups and possibly systems) involved [3]. This model is well suitable to describe collaboration support systems that fulfil the requirements above.

\section{Collaboration Management}

Recent studies show that success of knowledge sharing is dependent on the level of trust and dependency between community members and on the kind of culture holding in the society [2]. Technology can facilitate knowledge sharing, but it is trust that enables it. Sharing knowledge therefore implies that seekers and owners must be able to find each other and agree on the terms of the exchange. Moreover, the value of a knowledge item cannot be fixed a priori but depends on many factors, and knowledge and information requests cannot be fulfilled by a mere exchange of finished 'products' but require an, often not trivial, process during which the knowledge owner will develop the answer sought by the requester. Moreover, there must be a reciprocity in exchanges. In co-located groups, an exchange of favors relies on the assumption of stability of the community or group cohesiveness. A member of the community may provide information needed by another member because of a sense of community. There may be an inherent expectation that since the relationships 
within the community are typically long lasting, sooner or later the favor is likely to be returned. However in distributed groups, although the common goal binding the members remains long-term, contacts and relationships may be relatively fluid with members entering and exiting as their task needs evolve. In this scenario, exchange of favors is likely to be based on reciprocity in a relatively short time-span. That is, collaboration will need to be based in concrete, explicit commitments making clear what each partner is supposed to contribute and expects from the others.

\section{A Scenario for Collaboration and Knowledge Sharing}

KennisNet is a project taking place at Achmea which objectives are to structure, initiate and organize the sharing of knowledge between non-life insurance experts across Achmea by setting up a framework that assures the continuous availability of consistent and up-to-date knowledge [4]. Members of KennisNet are active across business units and are not part of any existing organizational structure.

In order to facilitate the creation of trust across the group, a dual approach for the development of KennisNet was chosen, which combines direct contacts between members of the group with a intranet-based knowledge sharing server. Direct contacts between participants were formalized as quarterly workshops attended by all members. In one hand, these workshops assure the creation, maintenance and uniformity of domain knowledge (for example, through talks by invited experts and facilitation of structured discussions around a theme), and on the other hand, the workshops contribute to the development of community feeling among the group.

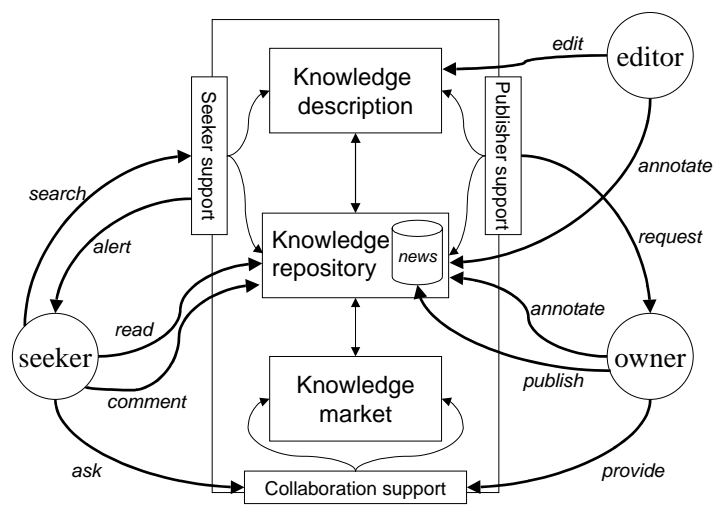

Fig. 1. The architecture of KennisNet

Parallel to the workshops, a knowledge sharing server was developed, whose architecture is depicted in figure 1. During the first phase the knowledge description and knowledge repository components were implemented based on the existing technical infrastructure, a Lotus Notes network. The functionality of Lotus Notes was used to support direct access to contents, as well as publishing and browsing of knowledge items. This infrastructure, inspired by work on knowledge repositories and organizational memories (e.g. [7,10]) allows for the implementation of facilities for discussion and broadcast of questions and requests. We have conducted a user satisfaction survey after the system was running for one year. The two main conclusions from this survey are that the workshop structure is greatly appreciated 
and found of great value but the added value and potential of the knowledge server is not clear to the users and the server is hardly used. The main reason for this lack of use, as pointed in the survey, is that users need a more personal means of interaction to make them comfortable exchanging knowledge. The survey also indicates that knowledge owners prefer to share their expertise within a controllable, trusted group under conditions negotiated for the specific situation and partners. That is, users wish to keep the decision about sharing knowledge on their own hands, and want to be able to decide on a case by case basis whether an exchange is interesting to them or not, which is also explained by the need for reciprocity in knowledge exchange [1].

People will agree on sharing their knowledge with others if they feel that they will gain something from the exchange. For example, I will share the result of a market survey I've just done with you, if you will let me have a copy of the report you are making from which you want to have those results. Therefore, a knowledge sharing system must be able to handle the negotiation and realization of this kind of agreements. We have chosen a agent-based approach for Knowledge Market, the extension of KennisNet that will enable personalized knowledge sharing. In one hand agents ensure the preservation of individual needs and perspectives and on the other hand are employed to monitor and assist on the exchange. That is, the moment an agreement as in the example above is made, the personal assistants of the partners, will take care that deadlines are kept, that reports are effectively exchanged, that eventual changes are communicated, etc. Furthermore, agents are used to search the network for suitable partners, to publish and search results in the repository on behalf of their owners, and to monitor news and discussion groups.

The Knowledge Market that will be described in detail in section 4, was developed according to the Agent Society Model that is introduced in section 3. It adds the following functionality to KennisNet:

- $\quad$ Possibility to share knowledge that is not available in the knowledge repository

- Support for coalition formation (in order to develop new solutions when knowledge is not available)

- Support for direct exchange between parties where the negotiation of exchange conditions happens in a case to case basis.

\section{The Agent Society Model}

The Agent Society framework distinguishes between the mechanisms though which the structure and global behavior of the model is described and coordinated, and the aims and behavior of the service-providers (agents) that populate the model [3]. The framework emerges from the idea that in organizations interactions occur not just by accident but aim at achieving some desired global goals. That is, there are goals external to the individual participants that must be achieved through the interaction of those participants. In the framework, interaction between agents is represented in a way that (1) is independent of the internal design of the agents, and (2) integrates organizational characteristics and demands with agent's own goals in a dynamic way that preserves the autonomy of the participating agents. Contracts are used to combine top-down specification of organizational structures with the autonomy of participating 
agents. Currently several related approaches, that take an organizational perspective on the development of multi-agents systems, are been developed [8,9].

The framework consists of three interrelated models. The organizational structure of the society, as intended by the organizational stakeholders, is described in the Organizational Model (OM). The OM specifies an agent society in terms of four structures: social, interaction, normative and communicative. The social structure specifies objectives of the society, its roles and the model that governs coordination. The interaction structure gives a partial ordering of the scene scripts that specify the intended interactions between roles. Society norms and regulations are specified in the normative structure, expressed in terms of role and interaction norms. Finally the communicative structure, specifies the ontologies for description of domain concepts and communication illocutions. The way interaction occurs in a society depends on the aims and characteristics of the application, and determines the way roles are related with each other, and how role goals and norms are 'passed' between related roles. For example, in a hierarchical society, goals of a parent role are shared with its children by delegation, while in a market society, different participants bid to the realization of a goal of another role.

The agent population of an OM is specified in the Social Model (SM) in terms of social contracts that make explicit the commitments regulating the enactment of roles by individual agents. Social contracts describe the capabilities and responsibilities of an agent within the society, that is the desired way that an agent will fulfil its role(s). The use of contracts to describe activity of the system allows in one hand for flexibility in the balance between organizational aims and agent desires and on the other hand for verification of the outcome of the system. Finally, given an agent population for a society, the Interaction Model (IM) describes possible interaction between agents. At this level all the characteristics and requirements of the society are incorporated in the software agents themselves. Agent will thus contain enough information and capabilities to interact with others according to the society specification.

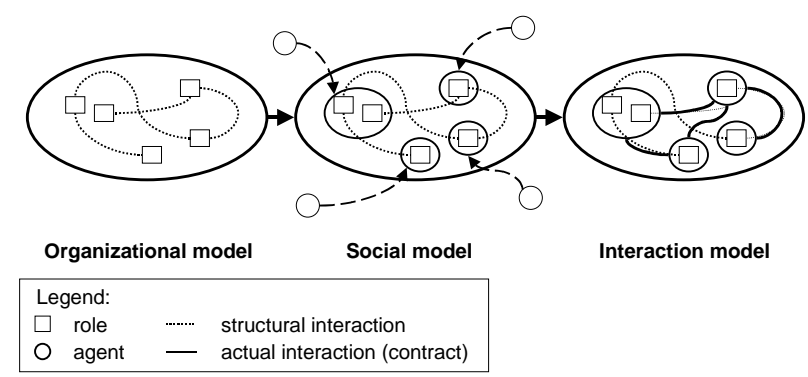

Fig. 2. Organizational framework for agent societies

Figure 2 depicts the relation between the different models. A generic methodology to analyze a given domain and determine the type and structure of the agent society that best models that domain is described in [6]. The methodology provides generic facilitation and interaction frameworks for agent societies that implement the functionality derived from the co-ordination model applicable to the problem domain. Standard society types as market, hierarchy and network, can be used as starting point for development and can be extended where needed and determine the basic norms 
and facilitation roles necessary for the society. These coordination models describe the different types of roles can be identified in the society and issues such as communication forms, desired social order and co-operation possibilities between partners. In the next section we take the development of the Knowledge Market as an example for the specification of the different levels of the Agent Society Model.

\section{Knowledge Market: An Agent Society for Knowledge Sharing}

The Knowledge Market aims to support people exchange knowledge with each other, in a way that preserves the knowledge, rewards the knowledge owner and reaches the knowledge seeker in a just-in-time, just-enough basis. The system is being developed using the Agent Society Model, and is described in the remaining of this section.

\subsection{Organizational Model}

Due to space limitations, and since the normative and communicative structures can be for the most part understood from the social and interactions structures, we will only describe the social and interaction structures in detail.

\subsubsection{Social Structure}

The global objective of the Knowledge Market is to support knowledge exchange. The requirements indicate the need for both direct exchange, directed at finding relevant partners, and indirect exchange, through the repository, in which case the task of the system is to support publishing the results of direct knowledge exchanges. The OM of an agent society consists of two layers: operation and facilitation and is dependent on the coordination model of the domain.

The Knowledge Market is characterized by informal relationships between independent partners, interested in collaborate in a win-win way. This, and other characteristics not discussed here, indicate that this community is coordinated according to the network type. This determines the following facilitation roles for the Knowledge Market [6]:

- Gatekeeper (GK): is responsible for accepting agents and fixing their social contracts. It must check whether an applying agent represents a member of the KennisNet group. The conditions and restrictions of agents concerning sharing of results related to their interaction with others are fixed in the social contract. Typically in the KennisNet, members do not have restrictions concerning sharing of knowledge they bring in. However, specially when new products are concerned it can happen that agents involved will require such knowledge to be shared only within a restricted group.

- Matchmaker (MM): keeps track of members, their needs and skills, and mediates the match of demand and supply². In the present situation, matching is done using keywords. The matchmaker presents a list of potential partners to the

\footnotetext{
2 The actual matching of supply and demand of knowledge is a very complex process that, due to space restrictions, will not be further discussed here.
} 
requesting agent. In the case that no (good) matches are available for a knowledge request, the matchmaker can decide to send a call to knowledge owners asking whether they are available and interested in that request.

- $\quad$ Notary (N): registers collaboration agreements (interaction contracts) between members. These contracts are necessary to monitor the activity of the society and the participation of members. Contracts also enable the proactive action of agents to support the interaction between the people involved. The notary is also responsible for imposing sanctions upon violators of contracts.

- Monitor (M): keeps track of the realization of contracts (deadlines and results) and takes care of delivering its results to the agreed recipients and publishes results for further use by other members of the community ${ }^{3}$. Monitors must also tell the notary about eventual violations.

Furthermore, the Knowledge Market OM must specify user roles, to be enacted by agents acting as avatars for KennisNet users. The sharing activities that characterize Knowledge Market require the presence of two roles:

- Knowledge Seeker (KS): represents needs of a user seeking collaboration. In the current approach, a knowledge seeker agent is created for each knowledge request. In principle, there can be several

- Knowledge Owner (KO): represents capabilities and interests of a user.

As described in [4], roles are specified in terms of goals and norms. For example, the role of knowledge seeker can be described has follows:

\begin{tabular}{|cl|}
\hline Goals & \multicolumn{1}{c|}{ Role: Knowledge Seeker } \\
Norms & acquire-knowledge(description-of-request, resulting-item) \\
1. & $\begin{array}{l}\text { Before placing a request, the seeker is required to first consult the KennisNet } \\
\text { repository (in order not to ask unnecessary work from others) }\end{array}$ \\
2. $\begin{array}{l}\text { Seekers will offer a retribution service within its means and according to the } \\
\text { interests of the knowledge owner as 'payment' for received services }\end{array}$ \\
3. Seekers will make the knowledge received as result of an exchange available to \\
others through the repository (within its sharing and privacy constraints)
\end{tabular}

For the sake of readability, norms, goals and landmarks are described throughout this paper in an informal and intuitive way. In the OM, this is formalized and made operational using the formal language LCR [5].

\subsubsection{Interaction Structure}

An interaction structure describes the transitions between interaction scenes. An interaction scene script describes a scenario of activity, that is, how roles interact and evolve in the context of a scene. Interaction structures are depicted as directed graphs where the boxes represent scenes and the arcs possible transitions between scenes. Facilitation roles active in a scene are represented by an oval linked to the scene box.

\footnotetext{
${ }^{3}$ Publication of interaction results is done according to the publication rules (basically, who can access it, who cannot, etc.) specified in the social contract of the agents.
} 


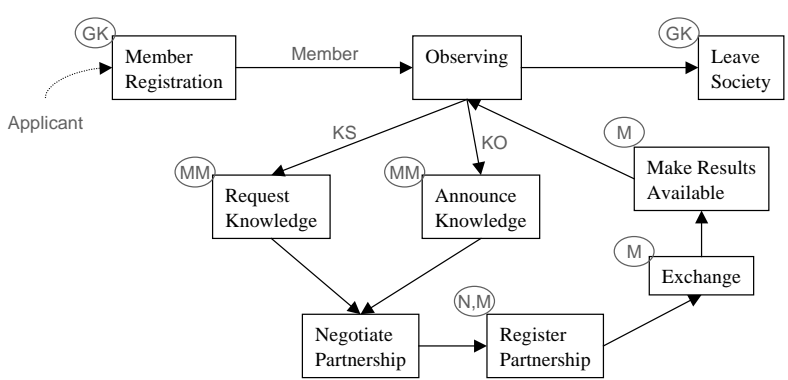

Fig. 3. Interaction structure of Knowledge Market

The interaction structure displayed in figure 3 describes the activity of the user roles (knowledge owner and seeker) in the Knowledge Market. Knowledge seekers and knowledge owners apply enter the society through the 'Member registration' scene. If the application is successful, the agent proceeds to the 'observing' scene. In this scene the agent is not actively active in a knowledge exchange but can access the repository, follow newsgroups, etc. Both seeker or owner agents can initiate an exchange by respectively announcing a need or a skill. In the 'negotiate partnership' scene, seeker and owner discuss the conditions of an exchange. The result is a interaction contract that describes an instance of the 'exchange' scene.

Interaction scripts serve as a blueprint for the actual interactions between agents enacting roles. Landmarks are logical expressions that describe the characteristics (for instance, goals and action plans) of the scene. Landmarks are a very flexible mechanism to specify interaction. Landmarks specify families of protocols, which do not need to be fixed at OM. The level of specification of landmarks determines the degree of freedom the actors have about their performance. In the Interaction Model, concrete interactions will be specified in interaction contracts that describe the actual protocols. Due to space limitations, it is not possible to describe each interaction scene script. We use the 'Request Knowledge' scene as an example. The informal specification of this scene script is as follows:

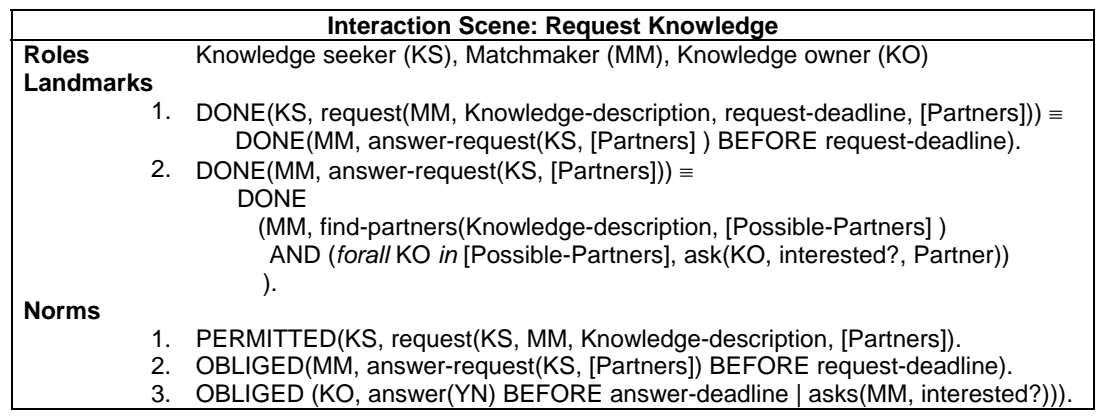

\subsection{Social Model}

In the Social Model, the action of independent agents in the society is specified. Such agents seek to enact one of the operational roles in the society. In the Knowledge Market, agents enacting a facilitation role are fixed in number and capabilities and are controlled by the society. Therefore, external agents cannot apply to a facilitation role. 
This is not the case in a generic Agent Society, which allows for independence of facilitation roles. However, in most cases, society design will specify a number of institutional roles in order to keep control over the society in some way or another.

People seeking collaboration through the Knowledge Market initiate a personal agent that acts as their avatar in the system. This agent uses the preferences and conditions specified by the user to find appropriate partners and negotiate exchange terms. Depending on the specific task, the personal agent will take either the role of knowledge seeker or knowledge owner. Requirements concerning privacy, secrecy and competitiveness between brands and departments that influence the channels and possibilities of sharing are also described in the specification of the personal assistants. Social contracts describe the agreements between participating agents and the Knowledge Market society. Negotiation of social contracts is done between the applicant agent and the Gatekeeper agent, which will watch over the interests of the society itself. For example, Anne is a member of the KennisNet group that is seeking knowledge on price policies from the concurrence. Anne will initiate an agent enacting the knowledge seeker role in the Knowledge Market. During the Member admittance scene, the conditions for Anne's agent will be negotiated and fixed in a social contract that specifies, for instance, which parts of the repository Anne is allowed to access, which are the obligations of Anne concerning the publication of knowledge items received as result of an interaction, and whether Anne allows for items that she provides to be published or not:

\begin{tabular}{|rl|}
\hline \multicolumn{2}{|c|}{ Social Contract: 'Anne-ID ' } \\
\hline Clauses & \\
1. & PERMITTED( Anne, access-kb([KB1, KB3, KB7]) \\
2. & OBLIGED(Anne, publish-received-knowledge(item, KB3) |allows(KO, publish)) \\
3. & allows(Anne, publish(item-provided-by-Anne, kb-of-receiver's-group)) \\
\hline
\end{tabular}

\subsection{Interaction Model}

The IM specifies the interaction agreements between role-enacting agents currently holding in the system. These agreements are specified using the contract language LCR, described in [5]. LCR allows the verification of society goals from the agreements specified in the contracts holding in the society.

The following example describes a contract between to members. In this example, fictive but typically possible in the domain of non-life insurance, Anne will provide Bob with a report about concurrent prices, on the condition that Bob will give her comments on the report (that she will have to present to her Unit directors) and eventually share with her his new pricing concept for car insurance. This contract is generated during the 'Negotiate partnership' scene and registered in the 'Register partnership' scene. In this scene, the notary agent will assign a monitor agent to check the fulfillment of the contract between Anne and Bob. Monitoring can be a very simple activity, where status is checked when a deadline is reached. However, we have chosen to use an agent as monitor because monitors can take an more active role, reminding parties of approaching deadlines or by suggesting possible actions when sanctions occur. The clauses of this contract are informally specified as follows:

\begin{tabular}{|ll|}
\hline \multicolumn{1}{|c|}{ Interaction Contract: 'ID' } \\
\hline $\begin{array}{l}\text { Parties } \\
\text { Clauses }\end{array}$ & Anne $(\mathrm{A})$, Bob (B) \\
\hline
\end{tabular}


1. OBLIGED A TO receive(B, report-concurrent-prices) BEFORE next-week

2. IF received(B, report-concurrent-prices) THEN

OBLIGED B TO

( receive(A, comment-report-concurrent-prices) BEFORE 3-days ) AND receive(A, concept-pricing) BEFORE 1-month

3. IF delayed(B, concept-pricing) THEN OBLIGED B TO inform(A, delayed(concept-pricing) )

In the case that either one of the agents will not fulfil its commitments, sanctions will be applied. When sanctions are not explicitly specified in the contract, the norms of the society will be used. For instance, the Knowledge Market follows the norm that agents that do not fulfil their commitments are given less priority in exchanges. Also it is possible to consider the publication of a list of best and worse members.

\section{Conclusions and Future Work}

Current developments in KM show a shift in the focus of KM from knowledge to collaboration. The aim of KM is no longer just the management of activities related to the creation, preservation and distribution of knowledge assets but the management and nurturing of collaboration between people. Such collaboration management systems call for approaches that are reactive and proactive in relation to the needs and expectations of its users. Agent concepts hold great promise for responding to the new realities of knowledge and collaboration management. In this paper, we have presented an agent-based model for organizations that fulfills the specification requirements of collaboration management systems. The model is being applied to the development of the Knowledge Market system at Achmea.

Agent concepts can fundamentally alter the nature of KM both in the way KM systems are build as well as the way organizations are analyzed and modeled. On one hand, the technical embodiment of these concepts can lead to advanced functionality of KM systems, e.g. personalization of knowledge presentation and matching supply and demand of knowledge. On the other hand, the rich representational capabilities of agents as modeling entities allow faithful and effective treatments of complex organizational processes. In our opinion, one of the main contributions of agent-based modeling of $\mathrm{KM}$ environments is that it provides a basis for the incorporation of individual initiative and collaboration into formal organizational processes.

\section{References}

[1] Ahuja, M., Carley, K.: Network Structure in Virtual Organizations. Journal of Computer Mediated Communication, 3(4), June 1998.

[2] Ali, I., Pascoe, C., Warne, L.: Interactions of organizational culture and collaboration in working and learning. IEEE Journal Educational Technology \& Society. 5(2), April 2002.

[3] Dignum, V., Meyer, J-J., Weigand, H., Dignum, F.: An Organizational-oriented Model for Agent Societies. In: Proc. Int. Workshop on Regulated Agent-Based Social Systems: Theories and Applications (RASTA'02), at AAMAS, Bologna, Italy, July, 2002.

[4] Dignum, V.: A Knowledge Sharing Model for Peer Collaboration in the Non-Life Insurance Domain. In: Proc. German Workshop on Experience Management, Lecture Notes in Informatics, German Society for Informatics, Berlin, 2002. 
[5] Dignum, V., Meyer, J.-J., Dignum, F., Weigand, H.: Formal Specification of Interaction in Agent Societies. $2^{\text {nd }}$ Goddard Workshop on Formal Approaches to Agent-Based Systems (FAABS), Maryland, October 2002.

[6] Dignum, V., Weigand, H.: Towards an Organization-Oriented Design Methodology for Agent Societies. In: V. Plekhanova (Ed.), Intelligent Agent Software Engineering. Idea Group Publishing, 2003.

[7] Domingue, J. B. and Motta, E.: A Knowledge-Based News Server Supporting OntologyDriven Story Enrichment and Knowledge Retrieval. Proc. EKAW'99, 1999.

[8] Esteva, M., Rodriguez, J., Sierra, C., Garcia, P., Arcos J.: On the formal specifications of electronic institutions, In Dignum F., Sierra C. (Eds.): Agent-mediated Electronic Commerce, LNAI 1991, pp. 126-147, Springer, 2001.

[9] Ferber, J., Gutknecht, O.: A meta-model for the analysis and design of organizations in multi-agent systems. Proc. of ICMAS’98, IEEE Press, 1998.

[10] Gandon, F., Dieng, R., Corby, O. \& Giboin, A.: A Multi-Agent System to Support Exploiting an XML-based Corporate Memory. Proceedings PAKM'00, Basel, 2000. 